\title{
Generalizations of MICZ-Kepler system
}

\author{
Armen Nersessian
}

Yerevan State University, 1 A.Manoogian St., Yerevan, 375025 Armenia

Artsakh State University, , Stepanakert \& Yerevan Physics Institute, Yerevan, Armenia

\begin{abstract}
We discuss the generalizations of the MICZ-Kepler system (the system describing the motion of the charged particle in the field of Dirac dyon), to the curved spaces, arbitrary potentials and to the multi-dyon background.
\end{abstract}

The integrable system describing the motion of the charged particle in the field of Dirac dyon (magnetic monopole carrying the electric charge) has been suggested independently by Zwanziger [1] and by McIntosh and Cisneros [2] and presently is known as MICZ-Kepler system. It is defined by the following Hamiltonian

$$
\mathcal{H}_{\mathrm{MIC}}=\frac{\pi^{2}}{2 \mu}+\frac{s^{2}}{2 \mu r^{2}}+\frac{\alpha}{r}, \quad \text { where } \quad\left[\pi_{i}, \pi_{j}\right]=-s \frac{\varepsilon_{i j k} x_{k}}{r^{3}}, \quad\left[\pi_{i}, x_{j}\right]=-\imath \delta_{i j}
$$

The distinguished peculiarity of the MICZ-Kepler system is the close similarity with Coulomb problem, which lie in the existence of a hidden symmetry given by the angular momentum operator $\mathbf{J}$ and by the analog of Runge-Lenz vector, which are defined by the expressions

$$
\mathbf{J}=\mathbf{r} \times \boldsymbol{\pi}+s \frac{\mathbf{r}}{r}, \quad \mathbf{A}=\frac{1}{2 \mu}[\boldsymbol{\pi} \times \mathbf{J}-\mathbf{J} \times \boldsymbol{\pi}]+\alpha \frac{\mathbf{r}}{r} .
$$

This hidden symmetry exists due to the appearance, in the Hamiltonian, of the specific centrifugal term $s^{2} / 2 r^{2}$. The origin of the additional centrifugal term $\frac{s^{2}}{2 \mu r^{2}}$ can be understood as follows [3]: In the presence of monopole magnetic field the angular momentum of the system gets additional, spin-like term $s \mathbf{r} / r$. The magnetic field of Dirac dyon is $\mathbf{B}=g \mathbf{r} / r^{3}$. On the other hand, there is linear relation between orbital and magnetic momenta of single particle, $\mathcal{M}=\frac{e}{2 \mu c} \mathbf{J}$. Hence, the interaction energy of this magnetic momentum with magnetic field $\mathbf{B}$ is given by the expression:

$$
U_{B}=-\mathcal{M} \mathbf{B}=-\frac{e}{2 \mu c} \mathbf{J} \frac{g \mathbf{r}}{r^{3}}=\frac{s^{2}}{2 \mu r^{2}},
$$

i.e. coincides with the centrifugal term in the MICZ-Kepler system [3. Though, the additional part of the orbital momentum proportional to $s$ should rather be assigned to the electro-magnetic field than to the particle, this non-correct interpretation nevertheless leads to the correct expression. Let us mention, that MICZ-Kepler system also describes the relative motion of two Dirac dyons with electric and magnetic charges $\left(e_{1}, g_{1}\right)$ and $\left(e_{2}, g_{2}\right)$ with $e_{1} g_{2}-e_{2} g_{1}=c s$. Similar to above mentioned case, the centrifugal term $s^{2} / 2 \mu r^{2}$ could be interpreted as the interaction energy of the induced dipole momentum with the dyon electric field plus interaction energy of the induced magnetic moment with magnetic field (here $\mu$ is the reduced mass). Notice, also, that this term yields, when we treat to obtain the MICZKepler system, similar to the Coulomb system, from the four-dimensional oscillator [4, 3. Let us mention, in this respect, that the Schroedinger equation for the MICZ-Kepler system is equivalent to the Schroedinger equation for the system of two well-separated BPS monopoles/dyons (which possesses the Coulomb symmetry) [5]. The quantum mechanical spectra of the MICZ-Kepler and Coulomb systems looks also quite similar to each other,

$$
\mathcal{E}=-\frac{\mu \alpha^{2}}{2 n^{2}}, \quad n=n_{r}+l+1, \quad n_{r}=0,1, \ldots
$$

The actual observable difference of the MICZ-Kepler system from the Coulomb one lies in the change of the range of validity of the total angular momentum from $l=0,1, \ldots$ to

$$
l=|s|,|s|+1, \ldots, \quad m=-l, \ldots, l
$$

where the monopole number $s$ takes (half-)integer values. This leads to the $(2|s|+1)$-fold degeneracy of the ground state with respect to the azimuth quantum number $m$.

In some sense, quantum MICZ-Kepler system could be viewed as an exited hydrogen atom. However, upon perturbation by external fields, it gets qualitative differences. For example, in the linear potential field $e \mathbf{E r}$ there is the linear Stark effect, similar to the hydrogen atom. However, in contrast with the latter one, the linear Stark 
effect completely removes the degeneracy on the azimuth quantum number [6, 7], in contrast with the hydrogen atom. Particularly, the ground state is given by the expression

$$
\mathcal{E}_{0}=-\frac{\mu \alpha^{2}}{2(|s|+1)^{2}}+\frac{|e \mathbf{E}|}{\mu \alpha} m \operatorname{sgn} s\left(|s|+\frac{3}{2}\right)
$$

Also, considering the dipole transitions in the MICZ-Kepler system under influence of planar electromagnetic wave, one can observe the change of selection rules. Namely, in the conventional spherically symmetric quantum mechanical systems the selection rules are given by the expressions (see, e.g. [8]) $m^{\prime}=m, j^{\prime}=j-1 ; \quad m^{\prime}=m \pm 1, j^{\prime}=$ $j \pm 1 \quad m^{\prime}=m \pm 1, j^{\prime}=j \mp 1$. While in the MICZ-Kepler system other transitions are also possible:

$$
m^{\prime}=m, j^{\prime}=j \quad m^{\prime}=m \pm 1, j^{\prime}=j .
$$

This is consequence of the violation of $P$-parity by the magnetic monopole, and is the general feature of the quantum mechanical systems with monopoles (see [9, 7] and the references in [10]). The specific effect of the choice of the Coulomb potential is hidden symmetry, essentially simplifying the analyses of the system. For example, it makes possible the separation of variables in few coordinate systems [11. Few years ago the generalizations of the MICZKepler system to the three-dimensional sphere [12] and two-sheet hyperboloid [13] were suggested: the properties of these systems were also found to be quite similar to the ones of the respective Coulomb systems. It was shown recently in our paper with L.Mardoyan and A.Yeranyan, that this similarity is not occasional, but is the general peculiarity of the spherically symmetric systems with monopoles [14. Namely, if we put the Dirac monopole in the center of spherically symmetric system, and simultaneously change the potential in accordance with the rule

$$
U(r) \rightarrow U(r)+\frac{s^{2}}{2 G(r) r^{2}},
$$

(where $d s^{2}=G(r) d \mathbf{r} d \mathbf{r}, r=|\mathbf{r}|$ ), then the functional dependence of the energy spectrum on (radial and orbital) quantum numbers will not be changed. The only change in the spectrum of such system is the lifting of the low bound of the range of validity of the orbital quantum number (and degeneracy of the ground state) from 0 to $|s|$.

It is deductive, for illustration of these phenomena, to consider the classical trajectories of the generic spherically symmetric systems with Dirac monopole,

$$
\mathcal{H}=\frac{\pi^{2}}{2 G}+\frac{s^{2}}{2 G r^{2}}+U(r), \quad \text { where } \quad\left\{\pi_{i}, \pi_{j}\right\}=-s \frac{\varepsilon_{i j k} x_{k}}{r^{3}}, \quad\left\{\pi_{i}, x_{j}\right\}=\delta_{i j}
$$

The angular momentum of the system is given by the expression (2), from which it follows

$$
\mathbf{J r}=s r, \quad \mathbf{J}^{2}=\boldsymbol{\pi}^{2} r^{2}-(\boldsymbol{\pi} \mathbf{r})^{2}=s^{2} .
$$

In order to find the trajectories of the system, it is convenient to direct the $x^{3}$ axis along the vector $\mathbf{J}$, i.e. to assume that $\mathbf{J}=J_{3} \equiv J$. Upon this choice of the coordinate system, we get, from the first equation

$$
\cos \theta \equiv \frac{x^{3}}{r}=\frac{s}{J}
$$

From the second expression in (10) we get

$$
\boldsymbol{\pi}^{2}=p_{r}^{2}+\frac{\mathbf{J}^{2}-s^{2}}{r^{2}}, \quad p_{r}=\frac{\boldsymbol{\pi} \mathbf{r}}{r}
$$

Taking into account this expression, we can represent the Hamiltonian (9) in the following form

$$
\mathcal{H}=\frac{p_{r}^{2}}{2 G}+\frac{\mathbf{J}^{2}}{2 G r^{2}}+U(r), \quad \text { where } \quad p_{r}=G \frac{d r}{d t}
$$

Also, we can introduce the angle

$$
\phi=\arctan \frac{x^{1}}{x^{2}}, \quad \frac{d \phi}{d t}=\frac{2 J}{G r^{2}},
$$

and observe, that the time evolution of $\phi$ and $r$, as well as the form of trajectory, $r=r(\phi)$ are independent on monopole number $s$. The only impact of the magnetic monopole is the rotation of the orbital plane on the finite angle 
$\cos \theta=s / J$ breaking the $P$-symmetry of the system, and the lifting of the lower bound of the orbital momentum (see Eq.(10) $)$,

$$
|\mathbf{J}| \geq|s| .
$$

Hence, following the rule (8), we can construct the "MICZ-analog" of any integrable spherically-symmetric system, and present its explicit classical and quantum mechanical solutions!

Nevertheless, the "invariant meaning" of the magic centrifugal term $s^{2} / 2 g r^{2}$ has remained unclear yet, so that we can't still construct the "MICZ-analogs" of the non-spherically symmetric, but, nevertheless, integrable systems. While numerous important mechanical systems have no spherical symmetry. Among them the most known are twocenter Kepler systems [8. Let us remind, that problem of particle moving in the field of two Coulomb centers (or two-center Kepler problem) was solved in the middle of XIX century by Jacobi. He established the integrability of two-center Kepler system and of its limiting case when one of the forced centers is placed at infinity which yields the homogeneous potential field, in elliptic and parabolic coordinates respectively. The generalization of this picture to case where Coulomb centers are replaced by dyons has been proposed in our recent paper with S.Krivonos and V. Ohanyan [15]. There we proposed the generalization of the MICZ-Kepler replacement (8) which can be used in case of $N$ Dirac monopoles,

$$
U(\mathbf{r}) \rightarrow \frac{1}{2 G}\left(\frac{s_{1}}{r_{1}}+\ldots+\frac{s_{n}}{r_{n}}\right)^{2}+U(\mathbf{r}),
$$

where $s_{i}=e g_{i}$, with $g_{i}$ be the magnetic charge of the $i$-th monopole located at the point with coordinates $\mathbf{a}_{i}$, and $r_{i}=|\mathbf{r}-\mathbf{a}|$.

This replacement has the following important features:

- The system (without monopoles) admitting separation of variables in elliptic/parabolic coordinates results in the separable two-center MICZ-system (16) with the Dirac monopoles placed at the foci of elliptic/parabolic coordinates.

- The system admits the $\mathcal{N}=4$ supersymmetric extension at the following choice of potential

$$
U_{0}=\frac{\kappa}{G}\left(\sum_{I} \frac{g_{I}}{\left|\mathbf{r}-\mathbf{a}_{I}\right|}+\mathbf{B}_{0} \cdot \mathbf{r}\right)+\frac{\kappa^{2}}{2 G} .
$$

The respective supersymmetric system has been constructed by Ivanov and Lechtenfeld in 2003 [16].

Hence, in the case of the Euclidean metric, $G=1$, this replacement leads the well defined two-center MICZ-Kepler system. Also, in Euclidean space such a multi-center MICZ-Kepler system admits the $\mathcal{N}=4$ supersymmetric extension, if the electric and magnetic charges of background dyons has the same ratio, i.e. obey trivial Dirac-Schwinger-Zwanziger quantization condition

$$
g_{i} q_{j}-g_{j} q_{i}=0 .
$$

In that case the potential of the Euclidean multi-center MICZ-Kepler system belongs to the class (17) (up to unessential constant).

However, this procedure fails in case of the multi-center Coulomb systems on spheres and two-sheet hyperboloids, when the conformal flat metric and the potential are defined, respectively, by the expressions

$$
d s^{2}=\frac{4 r_{0}^{2}(d \mathbf{r})^{2}}{\left(1+\epsilon r^{2}\right)^{2}} .
$$

and

$$
U(\mathbf{r})=e \phi_{q_{1}, \ldots, q_{N}}=e \sum_{i=1}^{N} q_{i} \phi\left(\left|\mathbf{r}-\mathbf{a}_{i}\right|\right) .
$$

Here

$$
\phi(r)=\frac{1}{2 r_{0}} \frac{1-\epsilon r^{2}}{r}
$$

is the Coulomb potential on the sphere $(\epsilon=1)$ and two-sheet hyperboloid $(\epsilon=1)$ [17.

The reason is the following: due to the existence of hidden symmetries given by Runge-Lenz vector the (one-center) Coulomb system on Euclidean space admits the separation of variables in spherical, elliptic and parabolic coordinate. The Coulomb system on (pseudo)spheres also has the hidden symmetries given by the analog of Runge-Lenz vector. 
Analogously to Euclidean case, this hidden symmetry is connected to the the separation variables in few coordinate systems. These coordinate systems turn to the spherical, elliptic and parabolic ones at the planar limit. The connection between spherical and Cartesian coordinates on the sphere is identical with that on the Euclidean space, whereas the discrepancy is appeared for the elliptic and parabolic ones (see, e.g., 18 and refs therein). Therefore, the statement of 15] concerning the separability of variables for "MICZ-extended" systems is no more valid for the Coulomb systems on spheres and two-sheet hyperboloids. Moreover, the potential of the multi-center Coulomb system on (pseudo)sphere, does not belong to the class (17), admitting the $\mathcal{N}=4$ supersymmetric extension.

For the construction of the well-defined generalization multi-center MICZ Kepler system on sphere and other rotationally invariant curved spaces, we have to understand, where the centrifugal term in (16) came out from.

For this purpose let us remind, that Coulomb potentials on the three-dimensional Euclidean space, sphere and pseudosphere are nothing else, but the rotationally invariant Green functions for the Laplasians defined by the corresponding metrics, $\Delta \phi_{C}=\delta(\mathbf{r})$. While the vector potential of the Dirac monopole is just one-form dual to this Green function. Explicitly,

$$
* d A_{D}=-d \phi_{C}, \quad \Rightarrow \quad \Delta \phi_{C}=\delta(\mathbf{r}), \quad \Delta \equiv * d * d+d * d *
$$

Taking into account the duality between vectors and one- and two-forms in the three-dimensional spaces, one can write down these expressions in the following way

$$
-\nabla \phi_{C}=\operatorname{rot} \mathbf{A}_{D}, \quad \Rightarrow \quad \Delta \phi_{C}=\delta(\mathbf{r}) .
$$

Let us write down the explicit $s o(3)$ invariant solutions of this equations on the so(3) invariant spaces. The (zero-, one- and two-)form fields are independent on the metric, hence, one has to choose

$$
d \mathbf{A}_{D}(\mathbf{r})=\frac{(\mathbf{r} \times d \mathbf{r}) \wedge d \mathbf{r}}{2 r^{3}}, \quad d \phi(r)=\frac{d \phi(r)}{d r} \frac{\mathbf{r} d \mathbf{r}}{r} .
$$

On the other hand the rotationally invariant metrics can always be represented in the conformal-flat form $d s^{2}=$ $G(r)(d \mathbf{r})^{2}$. Taking into account the conformal flatness of the metrics, we get, from (22)

$$
\frac{d \phi_{C}}{d r}=-\frac{1}{r^{2} \sqrt{G(r)}}, \quad \Rightarrow \quad \phi_{C}=-\int \frac{d r}{r^{2} \sqrt{G(r)}} .
$$

Particularly, for the Euclidean space one obtains $\phi_{C}=1 / r$, and for the sphere and two sheet hyperboloid (with the metrics (19) the potential given by (20).

Then we define the multi-center MICZ-Kepler system on the $s o(3)$ invariant space by the expression

$$
\mathcal{H}=\frac{\left(\mathbf{p}-e \mathbf{A}_{g_{1}, \ldots, g_{N}}\right)^{2}}{2 G}+\frac{e^{2} \phi_{g_{1}, \ldots, g_{N}}^{2}}{2}+e \phi_{q_{1}, \ldots, q_{N}},
$$

where $\mathbf{A}_{g_{1}, \ldots, g_{N}}=\sum_{i} g_{i} \mathbf{A}_{D}\left(\mathbf{r}-\mathbf{a}_{i}\right), \phi_{q_{1}, \ldots, q_{N}}=\sum_{i} q_{i} \phi_{C}\left(r_{i}\right)$.

When the electric and magnetic charges of background dyons obey the condition (18), or, equivalently, $q_{i} / g_{i}=e \kappa$, this Hamiltonian can be represented in the form

$$
\mathcal{H}=\frac{\left(\mathbf{p}-e \mathbf{A}_{g_{1}, \ldots, g_{N}}\right)^{2}}{2 G}+\frac{e^{2}\left(\phi_{g_{1}, \ldots, g_{N}}+\kappa / e\right)^{2}}{2}-\frac{\kappa^{2}}{2} .
$$

where $\operatorname{grad}\left(\phi_{g_{1}, \ldots, g_{N}}+\kappa / e\right)=-\operatorname{rot} \mathbf{A}_{g_{1}, \ldots, g_{N}}$. This Hamiltonian admits the $\mathcal{N}=4$ supersymmetric extension on the Euclidean space [16] and on the sphere [19, 21] (provided the unessential constant $\kappa^{2} / 2$ is omitted).

Actually, the bosonic part of the supersymmetric system on sphere constructed by Bellucci, Krivonos and Shcherbakov [19] was the key ingredient, which led us to postulate the Hamiltonian of multi-center MICZ-Kepler system on so(3) invariant spaces (26). In our knowledge, the supersymmetric extensions of the MICZ-Kepler system on the generic conformal-flat space are unknown. However, it seems, that we believe, that with the explicit component expressions for the supercharges of the system on sphere [21, we will be able to construct it in the Hamiltonian framework.

Notice, that in the case of one background dyon the MICZ-Kepler Hamiltonian on the sphere and two-sheet hyperboloid coincides with the MICZ-Kepler Hamiltonian constructed within the rule (16) up to unessential constant $\epsilon s^{2} / 4 r_{0}^{2}$. Hence, it is separable in the spherical coordinates, and in the modified elliptic and parabolic coordinates considered in Ref. [18. It seems, that two-center MICZ-Kepler system on sphere and hyperboloid is also separable in these coordinates. 
Let us conclude our consideration by the following speculation. The one-center MICZ-Kepler system describes not only the motion in the field of fixed dyon, but also the relative motion of two dyons in the centre-of-mass system. Similarly, by the use of the replacement (8) we can equip the two-body system with the magnetic charges, without changing the qualitative properties of the initial system. Similar phenomenon was observed since its inception in the Calogero model 22 describing the $N$ one-dimensional identical particles with pair-wise inverse-square and harmonic interaction: the energy spectrum of Calogero system coincides with the one of $N$ free harmonic oscillators, apart from the coupling dependent shift of the ground-state energy (for the recent review see [23). This structure of spectrum has led Calogero to suggest the possibility of a map between Calogero model and the system of decoupled oscillators, which has been found very recently [24] (see also [25]). Taking in mind the mentioned similarity between two-body MICZ-Kepler system and Calogero system, as well as the structure of multi-center MICZ-Kepler system, one can suppose, that the following system of $N$ interacting monopoles could be viewed as a three-dimensional generalization of the Calogero model,

$$
\mathcal{H}=\frac{1}{2} \sum_{I}\left(\mathbf{p}_{I}-\sum_{J} s_{I J} A_{D}\left(\mathbf{r}_{I}-\mathbf{r}_{J}\right)\right)^{2}+\frac{1}{2} \sum_{\substack{I, J, K \\ I \neq J, I \neq K}} \frac{s_{I J} s_{I K}}{r_{I J} r_{I K}}+\frac{1}{2} \sum_{I} \omega^{2} \mathbf{r}_{I}^{2} U,
$$

where $r_{I J}=\left|\mathbf{r}_{I}-\mathbf{r}_{J}\right|, s_{I J}=e_{I} g_{J}-e_{J} g_{I}$. But we didn't checked this claim up to now.

Acknowledgements. I am indebted to Sergey Krivonos, Levon Mardoyan, Vadim Ohanyan, Armen Yeranyan for collaboration in problems reflected in this paper. I am grateful to the Organizers of Colloquium on Integrable models and Quantum symmetry and personally Professor Cestmir Burdik for kind hospitality in Prague. This work is partially supported upported by the grants NFSAT-CRDF ARP1-3228-YE-04 and INTAS-05-7928

\section{References}

[1] D. Zwanziger, Phys. Rev. 176, 1480 (1968)

[2] H. McIntosh and A. Cisneros, J. Math. Phys. 11, 896 (1970).

[3] A. Nersessian and V. Ter-Antonian Mod. Phys. Lett. A9, 2431 (1994);

[4] T. Iwai, Y. Uwano, J. Math. Phys. 27, 1523 (1986).

I.M. Mladenov and V.V. Tsanov, J. Phys., A20, 5865 (1987); J. Phys., A32, 3779 (1999).

[5] G. W. Gibbons and N. S. Manton, Nucl. Phys., B274, 183 (1986) .

L. G. Feher and P. A. Horvathy, Phys. Lett. B183, 182 (1987) [Erratum-ibid. 188B, 512 (1987)].

[6] L. Mardoyan, A. Nersessian and M. Petrosyan, Theor. Math. Phys., 140, 958 (2004)

[7] L. Mardoyan et al, J. Phys. A40, 5973-5980(2007)

[8] L.D. Landau,E.M. Lifshitz, Quantum Mechanics, Oxford: Pergamon Press, 1977.

A. S. Davydov, Quantum mechanics, Nauka Publ., Moscow, 1973

[9] E. A. Tolkachev, L. M. Tomilchik, Y. M. Shnir, Yad. Phys. 38,541 (1983) ; J. Phys. G 14, 1 (1988)

[10] E. A. Tolkachev, L. M. Tomilchik, cond-math/0610213

[11] L. G. Mardoyan, G. S. Pogosyan, A. N. Sissakian and V. M. Ter-Antonyan, Quantum systems with hidden symmetry, MAIK Publ., Moscow, 2006

[12] V. V. Gritsev, Yu. A. Kurochkin, V. S. Otchik, J. Phys. A33, 4903-4910 (2000)

[13] A. Nersessian and G. Pogosyan, Phys. Rev. A63, 020103(R) (2001)

A. Nersessian, Phys. Atom. Nucl. 65, 1070 (2002);

[14] L. Mardoyan, A. Nersessian, A. Yeranyan Phys. Lett. A366, 30-35 (2007) 
[15] S. Krivonos, A. Nersessian and V. Ohanyan Phys. Rev. D75, 085002 (2007)

[16] E. Ivanov, O. Lechtenfeld, JHEP 0309, 073 (2003).

[17] E.Schrödinger, Proc.Roy.Irish Soc. 46,9 (1941) ; 46,183 (1941).

[18] A. A. Bogush, V. S. Otchik and V. M. Red'kov, arXiv:hep-th/0612178.

[19] S. Bellucci, S. Krivonos and A. Shcherbakov, Phys. Lett. B645, 299 (2007)

[20] A. Nersessian and V. Ohanyan, arXiv:0705.0727], to appear in Theor. Math. Phys

[21] S. Bellucci, S. Krivonos and V. Ohanyan, arXiv:0706.1469,

[22] F. Calogero, J. Math. Phys. 10, 2191(1969); ibid. 12, 419(1971)

[23] A. P. Polychronakos, J. Phys. A39, 12793(2006)

[24] N. Gurappa and P. K. Panigrahi, Phys. Rev. B59, R2490-R24493(1999)

[25] A. Galajinsky, O. Lechtenfeld and K. Polovnikov, Phys. Lett. B643, 221(2006) 